\title{
Medikamentenadhärenz in der Asthmatherapie - eine strukturierte Übersicht
}

\author{
Medication Adherence in Asthma Therapy - A Structured Review
}

Autoren

Institute
J. Fischer ${ }^{1}$, A. Wimmer ${ }^{2}$, J. Mahlich ${ }^{3}$

${ }^{1}$ Med. Klinik I, Abt. Pneumologie \& Schlafmedizinisches Zentrum, Krankenhaus Landshut-Achdorf

2 Janssen, Neuss

3 Janssen, Neuss und Universität Wien, Institut für Volkswirtschaft eingereicht 22.4.2013 akzeptiert nach Revision 28.5.2013

\section{Bibliografie}

Dol http://dx.doi.org/

10.1055/s-0033-1344242

Online-Publikation: 24.6.2013

Pneumologie 2013; 67: 406-414

(c) Georg Thieme Verlag KG

Stuttgart · New York

ISSN 0934-8387

\section{Korrespondenzadresse} Prof. Dr. med. Jürgen Fischer Med. Klinik I, Abt. Pneumologie \& Schlafmedizinisches Zentrum Krankenhaus Landshut-Achdorf Achdorfer Weg 3 84036 Landshut juergen.fischer@kh-landshutachdorf.de

\section{Zusammenfassung \\ $\nabla$}

Hintergrund: Asthma gehört zu den chronischen Erkrankungen mit der niedrigsten Adhärenz. Es soll dargestellt werden, welche Faktoren die Adhärenz beeinflussen, welche Messverfahren es gibt und mit welchen Maßnahmen sich die Adhärenz verbessern lässt. Außerdem werden ökonomische Aspekte schlechter Adhärenz diskutiert. Methode: Im Rahmen einer systematischen Literaturrecherche wurden 53 relevante und aktuelle Arbeiten identifiziert und aufbereitet.

Ergebnisse: Zu den Ursachen einer schlechten Adhärenz gehören Überzeugungen und Verhalten der Patienten, Komorbiditäten, hier besonders psychische Komorbiditäten, die Arzt-Patientenbeziehung, Medikation und andere Faktoren. Inwiefern adhärenzsteigernde Maßnahmen kosteneffizient sind, ist noch nicht eindeutig belegt.

Schlussfolgerungen: Da die Asthmakontrolle entscheidend von der Adhärenz abhängt, ist die Entwicklung neuer Adhärenzprogramme, die auf die Individualität des Patienten eingehen, nötig.

\section{Einleitung}

Asthma lässt sich medikamentös nicht heilen, in den allermeisten Fällen jedoch gut behandeln. Ein wichtiges Therapieziel ist die Asthmakontrolle. Damit will man die chronische Entzündung der Atemwege beherrschen und Komplikationen sowie Folgeschäden vermeiden. Die antientzündliche Langzeittherapie kann so die Belastbarkeit des Patienten und seine Lebensqualität verbessern. Trotzdem ist die Adhärenz vieler Asthmatiker gegenüber ihrer Therapie nicht besonders hoch.

In diesem Artikel gehen wir der Frage nach, welche Faktoren die Adhärenz beeinflussen, welche Messverfahren es gibt und mit welchen Maßnahmen sich die Adhärenz verbessern lässt. Außer-

\section{Abstract \\ $\nabla$}

Background The adherence level of Asthma therapy is low. We provide a literature overview about the determinants of adherence in asthma therapy, and potential options that lead to an improvement of adherence. We also discuss cost issues of poor adherence.

Methods: A systematic literature search was conducted and 53 articles were identified.

Results: The most prominent driving factors of adherence are patient beliefs, comorbidities, physician-patient relationship, and medication regimen. The cost effectiveness of adherence improving measures has yet to be shown.

Conclusion: As asthma control is primarily depended on adherence, cost effective adherence programs need to be developed that respond to patients' individual needs.

dem werden ökonomische Aspekte schlechter Adhärenz diskutiert.

\section{Material und Methoden \\ $\nabla$}

Eine Pubmed-Abfrage identifizierte Publikationen über die Adhärenz zu einer Asthmabehandlung in Deutschland. Es wurden folgende Stichworte ausgewählt: asthma, adherence, persistence, compliance, compliant, utilization, utility, cost, education, german. Der Suchstring lautete: asthma*[Ti] AND (adherence[Ti] OR persistence [Ti] OR compliance[Ti] OR compliant[Ti] OR utilization[Ti] OR utility[Ti] OR cost*[Ti] OR education [Ti]) AND german*. 
Diese Suche ergibt 85 Ergebnisse, wovon 53 relevant erschienen. Die Relevanz der Ergebnisse wurde anhand der Titel bewertet und, sofern möglich, den Kategorien Adherence, Compliance, Persistence, Utility, Utilization, Education und Costs zugeordnet. Dadurch reduzieren sich die anfänglich 85 Treffer auf letztlich relevante 53 [1 -53]. Der ursprüngliche Suchstring hat den Zusatz „german“ enthalten, da primär Aussagen über deutschen Versorgungkontext gemacht werden sollen. Per Handsuche wurden jedoch weitere wichtige und systematisch hochwertige Arbeiten identifiziert und ergänzt.

\section{Ergebnisse}

\section{Adhärenz - eine Begriffsbestimmung}

Definition - Unterschied zur Compliance

Die Weltgesundheitsorganisation WHO [1] definiert Adhärenz als das Ausmaß, in dem das Verhalten einer Person - zum Beispiel die Medikamenteneinnahme - mit den Empfehlungen übereinstimmt, die gemeinsam mit dem Therapeuten vereinbart wurden. Anders ausgedrückt kann Adhärenz als die Einhaltung der gemeinsam von Patient und Arzt gesetzten Therapieziele beschrieben werden.

Damit unterscheidet sich die Adhärenz von der Compliance, die als das Ausmaß definiert wird, in welchem der Patient die Anweisungen des Arztes einhält. Die Compliance geht also im Unterschied zur Adhärenz von einer passiven Patientenrolle aus [2].

Dimensionen der Adhärenz

Die Adhärenz wird von fünf verschiedenen Faktoren oder besser Dimensionen beeinflusst, die untereinander interagieren können [1]:

1. Soziale und ökonomische Faktoren (sozialer Status, Bildungsniveau, Arbeitslosigkeit, Analphabetismus, Zuzahlung, Alter, Krieg etc.)

2. Gesundheitsbezogene Faktoren (schlecht entwickeltes Gesundheitssystem, schlechte Arzt-Patienten-Kommunikation, inadäquate Sprechzeiten etc.)

3. Krankheitsbezogene Faktoren (Schwere der Erkrankung, Ausmaß der Behinderung (psychisch, physisch, sozial, beruflich), eingeschränkte Partizipation, Komorbidität etc.)

4. Therapiebezogene Faktoren (komplexes Behandlungsregime, Dauer der Behandlung (zu kurz, zu lang), kein schneller Behandlungserfolg etc.)

5. Patientenbezogene Faktoren (Ressourcen, Wissen, Glaube, Vorstellungen, Bedenken, Nebenwirkungen, Vorteile, Nachteile, Psyche etc.).

\section{Adhärenz messen - verschiedene Methoden}

Es gibt verschiedene Möglichkeiten, die Adhärenz zu messen $[3,4]$. Dazu gehören direkte Methoden, wie zum Beispiel die Beobachtung der Medikamenteneinnahme oder die Messung des Medikamentenspiegels oder biologischer Marker im Blut. - Tab. 1 fasst verschiedene Verfahren mit ihren Vor und Nachteilen zusammen, wobei es keine allgemein akzeptierte „Best practice“ gibt. So ist die Patientenselbsteinschätzung kostengünstig und praktikabel, führt aber tendenziell zu einer Überschätzung der Adhärenz. Daher sollte man unbedingt auf die Validität der verwendeten Methode achten [4].

Im Bereich Asthma wird als Patienten-Selbsteinschätzungs-Instrument häufig der Morisky-Fragebogen eingesetzt [54]. Der erweiterte Morisky-Score ( Tab. 2 ) besteht aus acht Fragen, die entweder mit Ja oder Nein beantwortet werden. Für jedes Nein gibt es einen Punkt. Es können maximal 8 Punkte erreicht werden, die als hohe Adhärenz bewertet werden. Bei einem Cutpoint von unter 6 Punkten ist die Reliabilität gut (Cronbachs alpha: 0,83). Die Sensitivität (tatsächlich Adhärente werden als adhärent eingestuft) beträgt $93 \%$, die Spezifität (tatsächlich Nicht-Adhärente werden als nicht-adhärent eingestuft) liegt bei 53\% [54]. Andere Fragebögen zur Messung von Adhärenz sind zum Beispiel der Adherence Estimator, der von McHorney entwickelt und validiert wurde [5]. Es handelt sich um eine Selbsteinschätzung, die anders als der Morisky-Fragebogen den Grad der Zustimmung (komplett, in der Regel, manchmal) beziehungsweise Ablehnung (manchmal, fast immer, immer) zu drei Fragen bewertet. Diese Fragen decken diejenigen Überzeugungen der Patienten ab, die in psychometrischen Tests am sichersten mit Nicht-Adhärenz korrelierten. Die Sensitivität für Non-Adhärenz liegt bei $88 \%$.

$\mathrm{Zu}$ den ausführlicheren Fragebögen gehört der MARS (Medication Adherence Report Scale for Asthma), der 10 Fragen enthält. Der Vergleich mit einem elektronischen Adhärenz-Messsystem ergab eine gute Korrelation, wobei in englischer und spanischer Sprache getestet wurde [6].

Ein Problem von Adhärenz-Messinstrumenten ist die geringe Übereinstimmung der Ergebnisse, wenn man verschiedene Instrumente einsetzt [7]. Dies hat eine Querschnittsstudie mit 139 Patienten mit verschiedenen chronischen Erkrankungen zeigen können. Dabei wurden die validierten Instrumente Brief Medication Questionnaire (BMQ), Medication Adherence Survey (MAS) und Medical Outcomes Study (MOS) mit Rezepteinlösungs-Daten aus Apotheken verglichen. Es fand sich bestenfalls eine moderate Korrelation zwischen den drei Selbsteinschätzungsinstrumenten und der Rezepteinlösung. Die exakte Messung der Adhärenz mit Fragebögen ist schwierig, Daten aus der Adhärenzforschung sind nicht ohne Weiteres miteinander vergleichbar. Eine mögliche

\begin{tabular}{|lll|}
\hline Verfahren & Vorteile & Nachteile \\
\hline Tablettenzählung & kostengünstig & \\
\hline Einlösung von Rezepten & objektiv & Überschätzung \\
\hline Wiegen von Dosieraerosolen & leicht durchführbar & keine Gewähr von Einnahme \\
\hline Apothekenrezepte zählen & leicht zugängig & keine Gewähr von Einnahme \\
\hline elektron. Methoden (z. B. Chip) & objektiv & Überschätzung \\
\hline allg. Patientenangaben & praktikabel und kostengünstig & kostenintensiv \\
\hline Patienteninterview & kostengünstig & Überschätzung \\
\hline Patiententagebuch & Kostengünstig & Überschätzung \\
\hline Medikamentenspiegel & Bestätigung der Einnahme & $\begin{array}{l}\text { Abhängig von versch.Faktoren wie z. B. Ernährung, } \\
\text { Rauchen, Zeitpunkt der Einnahme etc. }\end{array}$ \\
\hline klinische Einschätzung & schnell und kostengünstig & Über- oder Unterschätzung \\
\hline
\end{tabular}

Tab. 1 Vor- und Nachteile verschiedener Adhärenz-Messinstrumente.

Quelle: nach Gillissen 2007 [4] 


\begin{tabular}{|c|c|c|}
\hline Frage & Nein & Jedes Nein $=1$ Punkt \\
\hline \multicolumn{3}{|l|}{ Vergessen Sie manchmal die Einnahme Ihrer Medikamente? } \\
\hline \multicolumn{3}{|l|}{$\begin{array}{l}\text { In den letzten zwei Wochen: Gab es irgendwelche Tage, } \\
\text { an denen Sie Ihre Medikamente nicht genommen haben? }\end{array}$} \\
\hline \multicolumn{3}{|l|}{$\begin{array}{l}\text { Haben Sie jemals Ihre Medikamente nicht bzw. weniger } \\
\text { genommen ohne es Ihrem Arzt zu erzählen, weil es Ihnen } \\
\text { schlecht ging, wenn Sie die Medikamente nahmen? }\end{array}$} \\
\hline \multicolumn{3}{|l|}{$\begin{array}{l}\text { Vergessen Sie manchmal Ihre Medikamente mitzunehmen, } \\
\text { wenn Sie reisen oder das Haus verlassen? }\end{array}$} \\
\hline \multicolumn{3}{|l|}{ Haben Sie gestern Ihre Medikamente genommen? } \\
\hline \multicolumn{3}{|l|}{$\begin{array}{l}\text { Wenn Sie sich besser fühlen, stoppen Sie manchmal Ihre } \\
\text { Medikamenteneinnahme? }\end{array}$} \\
\hline \multicolumn{3}{|l|}{$\begin{array}{l}\text { Täglich Medikamente zu nehmen ist für einige Menschen } \\
\text { ein echter Nachteil. Hat es Sie jemals gestört bzw. genervt, } \\
\text { sich an Ihren Behandlungsplan zu halten? }\end{array}$} \\
\hline $\begin{array}{l}\text { Wie oft haben Sie Schwierigkeiten, daran zu denken, } \\
\text { Ihre Medikamente zu nehmen? }\end{array}$ & $\begin{array}{l}\text { nie }=1 \\
\text { sehr selten }=0,75 \\
\text { manchmal }=0,5 \\
\text { meistens }=0,25 \\
\text { immer }=0 \text { Punkte }\end{array}$ & \\
\hline \multicolumn{3}{|l|}{ Punkte insgesamt } \\
\hline & $\begin{array}{r}\text { Auswertungsschlüssel } \\
\text { hohe Adhärenz } \\
\text { mittlere Adhärenz } \\
\text { geringe Adhärenz }\end{array}$ & $\begin{array}{l}8 \text { Punkte } \\
6 \text { bis }<8 \text { Punkte } \\
<6 \text { Punkte }\end{array}$ \\
\hline
\end{tabular}

Tab. 2 Morisky-Fragebogen.

Quelle: Morisky et al. 2008 [54]

Erklärung ist der unterschiedliche Aufbau der verschiedenen Fragebögen, sowohl in der Länge als auch in der Ansprache der Patienten.

\section{Empirische Untersuchungen zur Adhärenz}

Über alle Krankheiten hinweg hält sich nur jeder zweite chronisch kranke Patient an die mit seinem Arzt vereinbarten Therapiemaßnahmen - mit einer großen Schwankungsbreite, die unter anderem von der Definition und dem Messinstrument abhängt; auch regionale und kulturelle Unterschiede spielen hier eine Rolle. Das gilt auch für Asthma ( $\bullet$ Tab.3).

Zwei Studien konnten identifiziert werden, die Asthmaadhärenz in Deutschland schätzen. Mühlig [15] spricht in seiner Arbeit noch von Compliance, die zwischen 14\% und 87\% liegt. Für die hohe Variabilität seiner Schätzungen ist die unterschiedliche Operationalisierung der Compliance-Definition verantwortlich.

Hier stellt sich die Frage, wie die Compliance definiert wird, z.B. über die Anzahl der Behandlungstage, die Einhaltung einer minimalen Wirkstoffdosis oder eine unterbrechungsfreie Einnahme an einer definierten Anzahl von Tagen. Definiert werden sollte auch, ab wie viel Prozent Abweichung man von Non-Compliance spricht und wie die Patienten klassifiziert werden.

So erhält man bei einer Patientenstichprobe mit demselben elektronischen Medikamenten-Compliance-Messgerät ganz unterschiedliche Compliance-Raten. Hier exemplarisch einige Ergebnisse: An 84,5\% aller Behandlungstage wurde mindestens eine Applikation registriert. Folglich lag der Anteil der Nicht-Behandlungstage bei nur 15,5\%. Allerdings war bei 15,0\% der Behandlungstage eine Überdosierung zu sehen, bei 36,9\% jedoch eine Unterdosierung. Nur ein Drittel der Patienten (32,5\%) schaffte es, mindestens einmal sieben Tage ohne Unterbrechung ihre Verschreibung korrekt einzuhalten.

Eine andere Möglichkeit, Rückschlüsse auf die Patientenadhärenz zu ziehen, ist die Abbildung der Versorgungsrealität von Asthmapatienten mithilfe von Krankenkassendaten. Hasford und Kollegen [16] konnten an 483.051 bayerischen Asthmapa- tienten - sie hatten mindestens zweimal in 2,5 Jahren die Diagnose Asthma bronchiale erhalten - zeigen, dass die Adhärenz vor allem unter inhalativen Kortikosteroiden (ICS) sehr gering ist. „Nur“ 61,4\% der Patienten wurden überhaupt medikamentös behandelt. Von ihnen bekam wiederum die Mehrzahl (65,1\%) nicht mehr als 90 definierte Tagesdosen (DDD) an Controller-Medikamenten in dem Untersuchungszeitraum von 365 Tagen. Nur

Tab. 3 Adhärenz chronischer Krankheiten und bei Asthma.

\begin{tabular}{|c|c|c|}
\hline $\begin{array}{l}\text { Weltweit } \\
\text { (Allgemein) }\end{array}$ & Weltweit (Asthma) & Deutschland (Asthma) \\
\hline \multirow[t]{2}{*}{$\sim 50 \%[1]$} & $30-70 \%$ USA [56] & 14-87\% (Mühlig 2001, 15) \\
\hline & $30-70 \%$ USA [9] & \\
\hline$\sim 50 \%[55]$ & 40 - 78\% Europa [10] & $\begin{array}{l}90 \text { DDD im Zeitraum von } \\
12 \text { Monaten: } \\
53,5 \% \text { (ICS/LABA) } \\
55,6 \% \text { (LABA) } \\
65,1 \% \text { (ICS) } \\
72,3 \% \text { (SABA) } \\
{[16]}\end{array}$ \\
\hline \multirow[t]{5}{*}{$\sim 50 \%[8]$} & $\begin{array}{l}43 \% \text { und } 28 \% \text { (Controller } \\
\text { Med.), Australien [11] }\end{array}$ & \\
\hline & $\begin{array}{l}33-88 \% \text { ICS Nord-Irland } \\
45 \% \text { orales Prednisolon [12] }\end{array}$ & \\
\hline & 43,9\% Dänemark [13] & \\
\hline & $52 \%$ ICS Großbrittanien [6] & \\
\hline & $\begin{array}{l}75 \% \text { elektr. Gemessen } \\
\text { Australien } \\
85 \% \text { Selbstbericht } \\
82 \% \text { Männner } \\
66 \% \text { Frauen } \\
\text { (ICS/LABA, Salmeterol/ } \\
\text { Fluticason) } \\
{[14]}\end{array}$ & \\
\hline
\end{tabular}

DDD: defined daily doses, definierte Tagesdosis; ICS: inhaled corticosteroid, inhalatives Kortikosteroid; LABA: long acting Betaagonist, langwirkender Betaagonist; SABA: short acting Betaagonist, kurzwirkender Betaagonist. 
Tab.4 Überzeugungen und Verhalten.

Einnahme und Adhärenz von 100 Patienten, die mit ICS und LABA
behandelt wurden
$77 \%$ versuchten Medikamente wie verordnet zu nehmen
33 \% nahmen ICS nur bei Symptomen
$72 \%$ wussten, dass ICS auch dann genommen werden sollte, wenn keine
Beschwerden vorhanden sind
$48 \%$ gefiel es nicht, ein ICS zu benötigen
$24 \%$ fanden es schwierig, sich selbst zu motivieren
$33 \%$ bejahten Nebenwirkung vom ICS
$46 \%$ befürchteten, abhängig von ICS zu werden
$14 \%$ sagten, Freunde oder Familie haben geraten, Medikamente häufiger
zu nehmen

Teilnehmer: TN, ICS: Inhalatives Kortikosteroid, LABA: langwirkender Betaagonist. nach Foster 2012 [14]

etwa $1 \%$ erhielten Medikamente für den gesamten Untersuchungszeitraum.

Auch in anderen europäischen Ländern ist die Adhärenz schlecht. Dies zeigt zum Beispiel eine retrospektive Querschnittsstudie aus Irland [12]. Nach einem Aufenthalt in einer Spezialklinik für schweres Asthma wurden die 182 Patienten sechs Monate lang weiter betreut. Ihre Therapie bestand aus inhalativer Kombinationstherapie (ICS plus langwirksame $\beta_{2}$-Agonisten [LABA]) und kurzwirksamen Beta-Agonisten, 51 Patienten erhielten orales Prednisolon. Die Adhärenz der Patienten wurde u. a. anhand der Rezepteinlösungen und der Plasmablutspiegel (Prednisolon, Cortisol) ermittelt und mit der ursprünglich verschriebenen Medikation verglichen. Etwa ein Drittel der Patienten lösten weniger als $50 \%$ der ICS-Rezepte ein. Diese erreichten auch signifikant schlechtere Werte auf dem Asthma-spezifischen Quality of Life Score (AQLQ). Nachdem anfänglich alle Patienten behauptet hatten, ihre zu inhalierenden Medikamente wie besprochen einzunehmen, gaben später $88 \%$ eine schlechte Therapieadhärenz zu. Von den Patienten, die mit oralem Prednisolon behandelt wurden, war fast die Hälfte (45\%) nicht adhärent. Insgesamt lag die Nicht-Adhärenz bei Frauen mit 42\% deutlich höher als bei Männern mit $23 \%$.

Eine dänische Studie [13] befragte 244 Patienten mittels Fragebogen im Internet. 43,9\% berichteten über Nicht-Adhärenz zu dem Medikamentenregime. Nur eine Frage vorher hatten sie angegeben, dass ihnen das Medikamentenregime bekannt sei. Dies zeigt indirekt, dass gute Kenntnisse nicht mit adhärentem Verhalten einhergehen müssen.

Die elektronische Messung der Adhärenz bei 53 Asthmapatienten, die inhalative Kortikosteroide einnahmen, ergab, dass die Patienten an 52 von 100 Tagen ihre Medikamente inhalierten [6].

\section{Ursachen für schlechte Adhärenz}

Es gibt eine Vielzahl verschiedener Faktoren, die mit der Güte der Adhärenz korrelieren beziehungsweise sie beeinflussen oder vorhersagen können. Die quantitative Bewertung dieser Faktoren ist-wenn überhaupt erfolgt-mit großer Schwankungsbreite behaftet. Dies ist unter anderem unterschiedlichen Studiendesigns und verschiedensten Messinstrumenten der Adhärenz geschuldet.

Die beschriebenen Gründe für Nicht-Adhärenz können nach verschiedenen Schemata kategorisiert werden. So existieren zum Beispiel die fünf Dimensionen der Adhärenz nach der WHO-Definition [1], oder es werden Behandlungs-, Arzt- und Patienten-bezogene Barrieren beschrieben [17] oder andere Ordnungssyste- me angewendet. Wir haben die in der Literatur gefundenen Faktoren unter folgenden praxisnahen Punkten dargestellt:

- Überzeugungen (beliefs) bei Asthma

- Komorbiditäten

- psychische Komorbiditäten

- Arzt-Patienten-Beziehung

- Medikamente

- Andere

\section{Überzeugungen}

Patienten haben in vielen Fällen ihre eigenen Ansichten und Überzeugungen, wie ein Medikament wirkt und ob oder wann es ihnen hilft. Diese stimmen häufig nicht mit dem medizinischen Wissensstand überein - und Ärzten fällt es schwer, solche Überzeugungen zu akzeptieren. Diese Überzeugungen beeinflussen die Adhärenz erheblich [14], daher sollten sie zunächst akzeptiert und nicht gleich als „falsch“ abgewertet werden. Denn dies erzeugt Widerstand, gewünscht wäre eher Veränderung der Überzeugung.

Wenn Patienten glauben, dass die inhalative Therapie mit Steroiden eine notwendige Asthmatherapie ist, sind sie häufiger adhärent $[14,18,19]$. Das ergab die Selbsteinschätzung mittels MARSFragebogen. Diese korreliert mit der elektronischen Messung der Adhärenz [6].

Ein aktuelle Studie [14] findet in einer Faktorenanalyse sieben signifikant mit adhärentem beziehungsweise nicht-adhärentem Verhalten verknüpfte Themen:

1. erkannte Notwendigkeit

2. Sicherheitsbedenken

3. Asthma-Chronizität/Medikamenteneffektivität

4. Rat der Familie/Freunde

5. Motivation und Routine

6. Leichtigkeit der Handhabung

7. Zufriedenheit mit Asthmamanagement

Von den 100 von Foster [14] beobachteten Patienten versuchten die meisten, ihre Medikation wie verordnet zu nehmen. Sie hatten, wie in Tab. 4 dargestellt, aber unterschiedliche Schwierigkeiten, adhärent zu sein.

Ponieman (2009) hat bei 261 Patienten mit geringem Einkommen, häufigen Klinikeinweisungen, Notfalleinweisungen, Intubation und Gebrauch oraler Kortikoide eine ICS-Adhärenz von etwa $70 \%$ - gemessen mit der MARS - ermittelt [19].

Die meisten Patienten (82\%) waren überzeugt davon, dass es wichtig sei, ICS bei Beschwerdefreiheit zu nehmen, obwohl viele Patienten Angst vor Nebenwirkungen (49\%) und Abhängigkeit (37\%) hatten. Die Adhärenz-Wahrscheinlichkeit war um den Faktor 4,15 erhöht, wenn die Patienten glaubten, ICS auch bei Beschwerdefreiheit nehmen zu müssen und um den Faktor 2,23, wenn sie angaben, sie seien vertraut mit ICS. Hingegen reduzierte sich die Adhärenz-Wahrscheinlichkeit auf etwa die Hälfte, wenn die Patienten Angst vor Nebenwirkungen hatten (OR 0,52) oder das Therapieregime als schwer einzuhalten beurteilten (OR 0,48).

\section{Komorbiditäten}

Eine schlechte Asthmakontrolle ist sowohl mit den Komorbiditäten Rhinitis und Rauchen als auch einer schlechten Adhärenz gegenüber der Therapie mit ICS assoziiert. Dies hat eine Umfrage in 85 englischen Allgemeinarztpraxen ergeben. Von insgesamt 4429 Fragebögen kamen 3916 komplett ausgefüllt zurück. Alle befragten Asthmapatienten wurden mit inhalativen Steroiden behandelt [20]. 


\section{Psychische Komorbiditäten}

Depressionen und Angststörungen sind häufige psychische Begleiterkrankungen bei chronisch kranken Menschen. Sie sind mit einem schlechteren Gesundheitszustand verknüpft ebenso wie mit höherer Nutzung des Gesundheitssystems. Eine Metaanalyse aus dem Jahr 2000 [21] fand einen signifikanten Zusammenhang zwischen dem Vorliegen einer Depression und der ermittelten Adhärenz mit einer Odds Ratio (OR) von 3,03. Das bedeutet, depressive Patienten haben eine dreifach höhere Wahrscheinlichkeit, nicht adhärent zu sein, als nicht depressive Patienten. Es war jedoch kein signifikanter Zusammenhang zwischen einer Angststörung und der Adhärenz zu sehen. In die Metaanalyse gingen 12 Depressions-Studien und 13 Studien zu Angststörungen bei verschiedensten chronischen Erkrankungen ein.

Auch eine prospektive Studie mit 102 Asthmapatienten [22], die mit ICS und LABA behandelt wurden, ergab für nicht-adhärente Patienten einen signifikant höheren Mittelwert im DepressionsScore (Hospital Anxiety and Depression Scale, HADS) als für adhärente Patienten $(4,7 \pm 3,3$ vs. $3,2 \pm 2,5)$.

Dass depressive Symptome mit einem deutlich höheren Risiko für Nicht-Adhärenz einhergehen, trifft auch für Patienten mit schwerem Asthma zu [23]. Die Patienten ( $\mathrm{N}=59)$ wurden wegen akuter Exazerbation ihres Asthma bronchiale stationär mit oralem Kortison und dem inhalativen Kortison Fluticason behandelt, die Medikamenteneinnahme wurde elektronisch und anhand des Cortisol-Blutspiegels überwacht. Bei der erneuten Vorstellung nach zwei Wochen waren „nur“ $60 \%$ der depressiven Patienten adhärent, während 74\% der Patienten ohne depressive Symptome ihre Medikation regelmäßig einnahmen. Nach Adjustierung möglicher beeinflussender Faktoren (Alter, Geschlecht, Schulbildung) ergibt sich für Patienten mit ausgeprägter Depression ein 11,4-fach höheres Risiko für schlechte Adhärenz, verglichen mit nicht-depressiven Asthmatikern [23].

Auch wenn man eher „extreme“ Patientensubpopulationen betrachtet - einmal Patienten mit einem sehr niedrigen Einkommen und hohen monatlichen Medikamentenkosten und umgekehrt Patienten mit sehr hohem Jahreseinkommen und niedrigen Medikamentenkosten - steigt in beiden Gruppen die Wahrscheinlichkeit für nicht-adhärentes Verhalten, wenn eine Depression vorliegt [24].

\section{Arzt-Patienten-Beziehung}

In einem älteren Übersichtsreferat differenzieren Niggemann und Wahn [25] zwischen Non-Compliance des Patienten und Non-Compliance des Arztes. Zwei wichtige Punkte betreffen das Arzt-Patienten-Verhältnis:

- missverständliche oder fehlende Instruktion des Arztes, im weitesten Sinne Defizite in der Kommunikation und - kein vertrauensvolles Arzt-Patienten-Verhältnis.

Eine gute Kommunikation zwischen Arzt und Patient ist eine wichtige Komponente des therapeutischen Prozesses. Sie stellt sicher, dass der Patient seine Erkrankung und die Behandlung versteht und die Medikamente so einnimmt, wie vom Arzt verschrieben. Wenn Patienten das Kommunikationsverhalten von Ärzten beurteilen [26], fehlen vor allem Fragen danach, wie sie ihr Asthma im Alltag (31\%) managen und ob es Probleme beim Asthmamanagement zu Hause (24\%) gibt. Die Ärzte hingegen schätzen sich als nonverbal aufmerksam ein (93\%) und als die interaktive Kommunikation in Gang haltend (93\%). Nur 30\% bzw. $33 \%$ berichteten, dass sie den Patienten helfen, Entscheidungskriterien für das Selbstmanagement ihres Asthmas zu entwickeln und den Behandlungsplan an ihre individuellen Bedürfnisse anzupassen [26].

Das Arzt-Patienten-Verhältnis hat sich in den letzten Jahren gewandelt vom eher paternalistischen hin zu einem partnerschaftlichen, auch wenn in der Regel wegen großer Informations- und Kompetenzunterschiede die Beziehung als asymmetrisch einzustufen ist. Zu der neuen Partnerschaftlichkeit gehört auch, eine gemeinsame Therapieentscheidung zu treffen (shared decision making).

Der Arzt soll dabei die Therapieziele und Präferenzen der Patienten erkennen und benennen, um im nächsten Schritt darauf abgestimmte Behandlungsmöglichkeiten vorzuschlagen und die jeweiligen Vor- und Nachteile - v.a. im Hinblick auf die individuellen Therapieziele und Vorlieben - zu diskutieren. Erst dann wird gemeinsam mit dem Patienten eine Entscheidung für ein Therapieregime getroffen. Ein solches Vorgehen führt zu einer deutlich besseren Adhärenz ebenso wie zu einer besseren Asthmakontrolle [27].

\section{Medikamente}

Auch die Medikation selbst, zum Beispiel die Häufigkeit der Einnahme und Wirkung der Medikamente beeinflusst die Adhärenz. Verzögerte und komplexe Therapieregime, Nebenwirkungen, Kosten und verzögerter Beginn der Behandlung wirken sich negativ auf die Adhärenz aus [17].

Andere medikamentenspezifische Einflussfaktoren sind [4]:

1. bessere Adhärenz mit Kombinationspräparaten (ICS und LABA in einem Inhaler) (2 Studien)

2. bessere Adhärenz mit Relievern als mit Controllern (2 Studien)

3. bessere Adhärenz mit Tabletten verglichen mit ICS (5 Studien)

4. kein Unterschied zwischen Tabletten und ICS (2 Studien).

Daraus ergibt sich folgende Patientenakzeptanz:

Tablette>inhalative Kombinationstherapie > Einzelinhalatoren.

Je mehr Medikamente und je mehr Einzeldosen verordnet werden, desto geringer wird die Adhärenz. Eine schlechte Adhärenz (oder Compliance) ist mit einer Über-, meist aber mit einer Unterdosierung der Asthmamedikation assoziiert. In diese Analyse gingen 11 Studien zur Therapieadhärenz bei Asthma bronchiale ein, die im Zeitraum von 1995 bis 2005 publiziert wurden.

\section{Andere Faktoren}

Alkoholexzesse, wie zum Beispiel Komasaufen, sind signifikant mit medikamentöser Nichtadhärenz ( $\mathrm{OR}=0,63)$ verknüpft, während - anders als in anderen Studien - eine eingeschränkte mentale Gesundheit zum Beispiel aufgrund von Depressionen oder anderen psychiatrischen Erkrankungen nicht mit Adhärenzproblemen assoziiert ist. Das ergab eine Sekundäranalyse des California Health Interview Survey [28]. Die Daten umfassen 3,2 Millionen Kalifornier mit diagnostiziertem Asthma bronchiale, von denen 1,7 Millionen Symptome aufwiesen. Weitere Prädiktoren für eine schlechte Adhärenz waren Übergewicht, jüngeres Alter (18-44 Jahre), „etwas“ College-Bildung, Rauchen und nicht in regelmäßiger medizinischer Betreuung.

Weitere Faktoren für schlechte Adhärenz - manchmal auch als schlechte Patientenmitarbeit bezeichnet [4] - sind Probleme, die Erkrankung und ihre Behandlung zu verstehen, sprachliche Probleme, Missverständnisse sowie falsche Anwendung des Inhalationssystems, Vergesslichkeit oder fehlendes Krankheitsgefühl [4]. Aber auch Schwierigkeiten bei der Terminvergabe, Behandlung durch verschiedene Ärzte, Desinteresse des Arztes, insuffizientes Vertrauen in den Arzt und geringe Motivation des Patienten, sein Verhalten zu ändern [17]. 


\section{Einfluss von Adhärenz auf Asthmakontrolle}

Der Zusammenhang zwischen Adhärenz (Compliance) und Asthmakontrolle ist gut belegt [29]. Eine Verbesserung der Adhärenz verbessert auch die Asthmakontrolle. Das zeigt eine prospektive, randomisierte Studie [27]. Wichtige Faktoren, die dazu beitrugen, waren Patientenschulung und regelmäßige persönliche oder telefonische Kontakte. Diese Aussage wird auch von Holgate und Kollegen [30] unterstützt. Sie haben zehn Schlüsselpunkte zur Verbesserung der Asthmakontrolle benannt. Einer davon lautet: „Menschen mit Asthma sollen dazu befähigt werden, teilzuhaben und auszuwählen, welche Versorgung sie wünschen." Dazu sollte man auch psychologische Faktoren und den Lebensstil betrachten, um diejenigen Faktoren zu identifizieren, die zur schlechten Kontrolle beitragen können. Weiter sind Patientenschulung sowie eine gute Aufklärung über Nutzen und Risiken der Asthmatherapie erforderlich. Auch sollten die Ärzte darauf hingewiesen werden, wie wichtig es ist, die Patienten in das Asthmamanagement gut einzubinden [30].

Clatworthy [20] konnte zeigen, dass eine schlechte Asthmakontrolle $(A C Q \geq 1,5)$ mit einer geringeren Adhärenz gegenüber der Therapie mit inhalativen Steroiden $(\mathrm{OR}=1,35)$ verbunden war. Die Evaluation eines Asthma-Disease-Management Programms für Medicaid-Kunden im Staat Washington der USA belegte einen deutlichen Rückgang des Asthmaschweregrads bei Patienten, die ein Jahr im Programm geblieben waren. Initial waren 25\% der Patienten als „schwer“ eingestuft wurden, nach einen Jahr waren es „nur“ noch 16\% mit dem Schweregrad „schwer“ [57].

\section{Instrumente zur Asthmakontrolle}

ACSS: Asthma Control Scoring System

ACT: Asthma Control Test

ATAQ: Asthma Therapy Asessment Questionnaire

LASS: Lara Asthma Symptom Scale (Kinder)

Das ACQ war der erste evaluierte und publizierte Asthmakontrolltest, er ist weit verbreitet und wird sowohl in der Forschung als auch in der Praxis eingesetzt. Er besteht aus sieben Items. Das ACQ stimmt u. a. mit den klinischen Charakteristika Tagessymptome, eingeschränkte Aktivität, nächtliche Symptome, Bedarf an Reliever-Medikation und Lungenfunktion $\left(\mathrm{FEV}_{1}\right)$ überein [31].

\section{Kosten durch schlechte Adhärenz}

Die ökonomischen Kosten für Asthma in Deutschland sind hoch und werden bereits für 1999 auf 2,7 Milliarden $€$ geschätzt, wobei knapp 1,2 Milliarden Kosten durch asthmabedingten Arbeitsausfall entstanden sind [58]. Diese Summe liegt nah an den von Nowak et al. [59] ermittelten 5,3 Milliarden DM(damals 3,1 Milliarden \$) für 1992. Die Autoren schlussfolgern, dass ein Großteil dieser Kosten durch eine bessere Asthma-Kontrolle eingespart werden könnte. Dies wird von anderen Autoren ebenso gesehen [60].

Die Kosten für einen Asthmapatienten liegen in den USA zwischen 2.646 \$ pro Jahr bei mildem Asthma über 4.530 \$ pro Jahr bei mittelschwerem Asthma bis zu 12.813 \$ pro Jahr bei schweren Asthmaformen. 35\% dieser Kosten sind durch Abwesenheit vom Arbeitsplatz und geringere Effektivität bedingt [32].

In Deutschland hatten im Jahr 2010 jedoch etwa 60\% der Patienten ihr Asthma nicht unter Kontrolle [61]. Deutschland scheint hier auch schlechtere Werte als andere europäische Länder aufzuweisen, Spanien z. B. weist für 2008 einen Wert von 47\% auf [62].

\section{Maßnahmen zur Verbesserung von Adhärenz}

Um die Adhärenz wirkungsvoll und nachhaltig zu verbessern, ist es wichtig, die Patienten mit schlechter Adhärenz zu erkennen. Zeichen für schlechte Adhärenz sind oben ausführlich dargestellt und bewertet.

\section{Verbesserung der Adhärenz allgemein}

Eine systematische Übersicht [33] hat 38 Reviews analysiert, die untersucht haben, wie effektiv verschiedene Interventionen die Adhärenz zu einer medikamentösen Therapie bei verschiedenen Erkrankungen verbesserten. 23 Reviews ergaben signifikante Unterschiede. In jedem der vier theoretisch anwendbaren Interventionsgebiete gab es deutliche Effekte:

- technische Interventionen: vereinfachte Medikation

- Verhaltensinterventionen: Patientenführung durch „reminder“ per Telefon, SMS etc.

- erzieherische Interventionen: Schulungen

- strukturelle Interventionen: DMPs

Dabei führten technische Lösungen wie die Vereinfachung des Therapieregimes zwar oft zu einer besseren Compliance, aber nicht immer. Interessant war auch, dass theoretische Modelle plausibel die Adhärenz zu verbessern scheinen, dies aber in der Realität nicht bewirken. Auf der anderen Seite lagen komplexen, aber wirksamen Interventionen keine besonderen Theorien zugrunde.

Ein Cochrane Review [55] konnte zeigen, dass 4 von 10 Interventionen in 9 randomisierten kontrollierten Studien sowohl die Adhärenz als auch mindestens einen klinischen Parameter verbesserte. Eine Intervention erhöhte zwar signifikant die Compliance, aber nicht die klinischen Outcome-Parameter.

Eine langanhaltende Verbesserung der Adhärenz wurde in 36 von 81 Interventionen in 69 randomisierten kontrollierten Studien beobachtet, aber nur 25 Interventionen besserten mindestens einen Behandlungsparameter.

Insgesamt waren alle Interventionen mit Langzeiteffekt komplex angelegt: aufwändige Betreuung, Information, Erinnerungen, Selbstbeobachtung, Beratung, Bekräftigung, Familientherapie, psychologische Therapie, Krisen-Intervention, Telefonanrufe und unterstützende Betreuung. Allerdings führte auch die effektivste Intervention von allen nicht zu einer großen Verbesserung der Adhärenz und Behandlungsergebnisse.

Arzt-Patienten-Verträge scheinen kein geeignetes Mittel zu sein, die Adhärenz bei Asthma langfristig zu verbessern, wie ein Cochrane Review zeigen konnte [34]. In die Auswertung gingen 30 Studien ein mit insgesamt 4.691 Patienten. Die Verträge wurden in den Bereichen Sucht (10 Studien), Hypertonie (4 Studien), Gewichtskontrolle (3 Studien) und anderen (13 Studien) geschlossen.

\section{Verbesserung der Adhärenz bei Asthma}

Die Patientenmotivation ist ein wichtiger Punkt, um die Adhärenz zu verbessern [17]. Dazu braucht es häufigere Patientenkontakte, Schulungen sowohl des Patienten als auch des Arztes und die Entwicklung eines partnerschaftlichen Verhältnisses zwischen Arzt und Patient.

Die gemeinsame Therapieentscheidung plus weitere Interventionen (Gruppe 1, N=182) führte zu einer deutlich besseren Adhärenz, verglichen mit einer „normalen“ Verordnung durch den Arzt plus weitere Interventionen (Gruppe $2, \mathrm{~N}=180$ ) oder einer 
sonst üblichen Behandlung (Gruppe 3, N=189). Dies zeigte eine prospektive, randomisierte Studie [27]. Bis auf die Modalität der Therapieentscheidung war das Vorgehen in Gruppe 1 und 2 identisch: Patientenschulung, 2 persönliche und 3 kurze telefonische Kontakte. Gruppe-3-Patienten erhielten die übliche Behandlung ohne zusätzliche Schulungen oder Gespräche. Nach 12-monatiger Behandlung hatten die Patienten, die gemeinsam mit dem Arzt ihre Therapie diskutiert und ausgehandelt hatten, folgende Vorteile gegenüber der Gruppe 2, deren Therapie durch den Arzt verordnet wurde:

- bessere Controller Adhärenz $(\mathrm{p}<0,03)$

- bessere LABA Adhärenz $(\mathrm{p}<0,014)$

höhere kumulative Controller Dosis $(\mathrm{p}<0,005)$

- quantitativ bessere klinische Asthmakontrolle (statistisch nicht signifikant)

Im Vergleich zu den Patienten, die eine übliche Behandlung ohne weitere Interventionen bekommen hatten, schnitten die an der Therapieentscheidung beteiligten Patienten noch besser ab:

- bessere Controller Adhärenz $(\mathrm{p}<0,0001)$

- bessere LABA Adhärenz $(\mathrm{p}<0,0225)$

- höhere kumulative Controller Dosis $(\mathrm{p}<0,0001)$

- geringere Inanspruchnahme des Gesundheitssystems und der Notfallmedizin

- bessere Asthmakontrolle ( $\mathrm{p}=0,002)$ und Lungenfunktion $\left(\mathrm{FEV}_{1}, \mathrm{p}=0,0068\right)$

- bessere asthmabezogene Lebensqualität $(\mathrm{p}=0,003)$.

Ein gutes Selbstmanagement kann die ICS-Adhärenz verbessern, wie eine 24-wöchige Studie mit 84 Patienten mit mittelschwerem bis schwerem Asthma zeigte [35]. Die Patienten bekamen entweder regelmäßige individualisierte Selbstmanagement-Unterweisungen zusätzlich zum Selbstmonitoring (Peak-Flow-Messung, Symptome notieren) oder nur eine Anleitung zum Selbstmonitoring.

Die Selbstmanagement-Unterweisung erfolgte alle vier Wochen und dauerte 30 Minuten. Inhalte waren: Asthma-Informationen, Überprüfung und Korrektur der Inhalationstechnik sowie ein individueller Plan für das Selbstmanagement und die Kontrolle von relevanten Umweltallergenen und irritativen Reizen. Die Patienten der Interventionsgruppe hatten eine 9-fach erhöhte Adhärenz, definiert als Inhalation von mehr als $60 \%$ der verschriebenen Dosis am Ende der Intervention. Am Ende der Studie war die Adhärenz für ICS immer noch 3-fach höher.

Auch bei schwer zu kontrollierenden Asthmapatienten wirkt eine auf mehreren Ebenen einsetzende Intervention. Zunächst wurden die nicht-adhärenten Patienten (83 von 239) anhand von Rezept-Einlösedaten identifiziert. Durch ein Konkordanzgespräch (Patient weiß, dass Arzt weiß ...) wurden 31 weitere Patienten adhärent. Gleichzeitig sank die benötigte Dosis an ICS, Rescue-Prednisolonbehandlungen und Krankenhauseinweisungen signifikant. In der zweiten Studienphase konnte die Adhärenz durch weitere protokollgemäße Interventionen noch verbessert werden [36].

\section{Strukturelle Intervention, am Beispiel der Disease}

Management Programme (DMPs)

Eine Strategie, die Adhärenz in der Asthmatherapie zu verbessern, sind Disease Management Progamme (DMPs). Innerhalb eines DMPs werden in der Regel folgende Instrumente angeboten [63]:

- Asthma-Schulungen

- Asthma-Tagebuch

- schriftliche persönliche Behandlungspläne
Tipps für die Praxis: Verbessern der Adhärenz

- Achte auf Marker für schlechte Adhärenz

- Frage nach Barrieren, ohne zu konfrontieren

- Betone Wichtigkeit der Behandlung und Adhärenz

- Ergründe die Gefühle des Patienten zur Therapie und seine Fähigkeit, dem Therapieregime zu folgen, unterstütze wenn nötig die Adhärenz

- Gib einfache, klare Anweisungen und vereinfache die Therapie soweit wie möglich

- Ermutige den Gebrauch eines Medikamenten-Einnahmesystems

- Versuche zusätzliche Unterstützung von Familienmitgliedern, Freunden und Gemeinde-Einrichtungen zu bekommen

- Höre auf den Patienten und erfülle seine Wünsche, so gut es geht

- Verstärke wünschenswertes Verhalten und Ergebnisse, so gut es geht

- Erwäge eher „vergebende“ Medikamente zu verordnen, wie Medikamente mit langer Halbwertszeit, Depot- oder transdermale Medikamente

nach Osterberg 2005 [3]

Allerdings verwenden nur zwischen 3\% und 33\% der Patienten persönliche Behandlungspläne [64,65], und es liegt eine geringe Bereitschaft zum Selbstmanagement vor [66]. 2008 waren in Deutschland 4,7 Millionen Patienten in DMPs eingeschrieben, 313.914 davon in ein Asthma DMP [67].

\section{Kosteneffizienz von Adhärenzmaßnahmen}

Zu der Frage, ob eine Verbesserung der Adhärenz in der Asthmatherapie auch ökonomisch wünschenswert ist, gibt es bislang nur wenige Aussagen.

Eine deutsche Studie zeigt, dass Teilnehmer eines Disease Management Programms (DMPs) zwar $300 €$ weniger stationäre Kosten verursachen, dafür aber $500 €$ höhere indirekte Kosten, die durch Arbeitsausfall verursacht werden. Aufgrund der geringen Fallzahl von 16 Patienten in dieser Gruppe sind die Ergebnisse allerdings mit Vorsicht zu interpretieren [68]. Aber durch die Teilnahme am DMP verbesserte sich die gesundheitsbezogene Lebensqualität signifikant.

Es gibt jedoch keine gesicherte Evidenz, dass DMPs bezüglich der Kosteneffektivität besser abschneiden als die Standardbehandlung $[69,70,71]$. Allerdings ist auch hier keine eindeutige Schlussfolgerung möglich, da etwa bei Diabetes-DMPs auch Kosteneinsparungen beobachtet wurden $[72,73]$.

Eine britische Studie aus den 90er-Jahren kommt zu dem Ergebnis, dass eine bessere Adhärenz bei Asthma nicht zu Kosteneinsparungen in anderen Bereichen des Gesundheitssystems führt, sondern nur zu einer Ausweitung der Arzneimittelkosten [74]. Neuere Daten [27] zeigen, dass eine signifikant bessere ICS- und LABA-Adhärenz die Asthmakontrolle verbessert und zu einer geringeren Inanspruchnahme des Gesundheitssystems und der Notfallmedizin führt. Die eingesparten Kosten für letztere Punkte sind nicht aufgeführt, die Kosten der Intervention werden jedoch abgeschätzt. Die Intervention in der Patientengruppe, die gemeinsam mit ihrem Arzt eine Therapieentscheidung traf und die besten Ergebnissen erzielte, beanspruchte etwa 3,2 Stunden Zeit und kostete etwa 179 \$. Die Kosten für die Standardbehandlung lagen bei $32 \$$. 


\section{Fazit}

Der gut belegte Zusammenhang zwischen Adhärenz und Asthmakontrolle unterstreicht, dass die Wirkung der inhalativen Asthmatherapie (vor allem) über die Adhärenz verbessert werden kann. Nur so kann letztendlich das Ziel der Asthmakontrolle erreicht werden.

Allerdings scheint es nicht ein Asthma Adhärenzprogramm für alle Patienten zu geben. Vielmehr zeigen die Daten, dass ein multimodales Vorgehen mit individuellen Angeboten für den Patienten die meisten Erfolge verspricht. In diesem Bereich sind weitere Untersuchungen nötig.

\section{Danksagung}

Wir danken Dr. rer nat. Carola Göring, Weilheim, für die Unterstützung bei der Bearbeitung des Manuskripts.

\section{Interessenkonflikt}

J. Fischer hat Vortragshonorare sowie Honorare für Beratertätigkeit von Janssen erhalten.

A. Wimmer und J. Mahlich sind Angestellte von Janssen.

\section{Literatur}

1 Sabaté E. Adherence to long-term therapies. Evidence of action. World Health Organization; 2003: 1- 194

2 Horne R. Compliance, Adherence and Concordance: implications for asthma treatment. Chest 2006; 130: 65S-72S

3 Osterberg L, Blaschke T. Adherence to medication. N Engl J Med 2005; 353: $487-497$

4 Gillissen A, Büsch K, Juergens U. Therapieadhärenz bei Asthma bronchiale, Definition - Einteilung - beeinflussende Faktoren. Dtsch Med Wochenschr 2007; 132: $1281-1286$

5 McHorney CA. The adherence estimator: a brief, proximal screener for patient propensity to adhere to prescription medications for chronic disease. Curr Med Res Opin 2009; 25: 215-238

6 Cohen JL, Mann DM, Wisnivesky JP et al. Assessing the validity of selfreported medication adherence among inner-city asthmatic adults: the Medication Adherence Report Scale for Asthma. Ann Allergy Asthma Immunol 2009; 103: 325-331

7 Cook CL, Wade WE, Martin BC et al. Concordance among three self-reported measures of medication adherence and pharmacy refill records. J Am Pharm Assoc 2005; 45: 151 - 159

8 Sackett DL, Haynes RB, Gibson ES et al. Patient compliance with antihypertensive regimens. Patient Couns Health Educ 1978; 11: 18-21

9 Rand CS, Wise RA. Measuring adherence to asthma medication regimens. Am J Respir Crit Care Med 1994; 149: 69-76

10 Cerveri I, Locatelli F, Zoia MC et al. International variations in asthma treatment compliance: the results of the European Community Respiratory Health Survey (ECRHS). Eur Respir J 1999; 14: 288 - 294

11 Reid D, Abramson M, Raven J et al. Management and treatment perceptions among young adults with asthma in Melbourne: the Australian experience from the European Community Respiratory Health Survey. Respirology 2000; 5: 281-287

12 Gamble J, Stevenson M, McClean E et al. The prevalence of Nonadherence in difficult asthma. Am J Respir Crit Care Med 2009; 180: 817 822

13 Møldrup C, Stein J, Søndergaard B. „Patients don't lie“; a view on adherence in asthma. Pharm World Sci 2010; 32: 795-798

14 Foster JM, Smith L, Bosnic-Anticevich $Z$ et al. Identifying patient-specific beliefs and behaviours for conversations about adherence in Asthma. Intern Med J 2012; 42: e136-144
15 Mühlig S, Petermann F, Bergmann K-CH. Verbreitung der Noncompliance bei Asthma-Patienten: Aktueller Forschungsstand und methodologische Probleme. Pneumologie 2001; 55: 163-176

16 Hasford J, Uricher J, Tauscher M et al. Persistence with asthma treatment is low in Germany especially for controller medication - a population based study of 483051 patients. Allergy 2010; 65: 347 - 354

17 Bender BG. Overcoming barriers to nonadherence in asthma treatment. Allergy Clin Immunol 2002; 109: 554-559

18 Emilsson M, Bendtsson I, Lötvall J et al. The influence of personality traits and beliefs about medicines on adherence to asthma treatment. Prim Care Respir J 2011; 20: 141 - 147

19 Ponieman $D$, Wisnivesky JP, Leventhal $H$ et al. Impact of positive and negative beliefs about inhaled corticosteroids on adherence in innercity asthmatic patients. Ann Allergy Asthma Immunol 2009; 103: $38-42$

20 Clatworthy J, Price D, Ryan D et al. The value of self-report assessment of adherence, rhinitis and smoking in relation to asthma control. Prim Care Resp J 2009; 18: 300-305

21 Dimatteo MR, Lepper HS, Croghan TW. Depression is a risk factor for Noncompliance with medical treatment. Arch Intern Med 2000; 160: $2101-2107$

22 Bosley CM, Fosbury JA, Cochrane GM. The psychological factors associated with poor compliance with treatment in asthma. Eur Respir J 1995; 8: 899-904

23 Smith A, Krishnan JA, Bilderback $A$ et al. Depressive symptoms and adherence to asthma therapy after hospital discharge. Chest 2006; 130 : $1034-1038$

24 Piette JD, Beard A, Rosland AM et al. Beliefs that influence cost-related medication nonadherence among the "haves“ and „have nots" with chronic diseases. Patient Preference and Adherence 2011; 5: 389-396

25 Niggemann B, Wahn U. Compliance bei Asthma bronchiale - ein Problem von Patient und Arzt. Pneumologie 1996; 50: 253-256

26 Newcomb PA, McGrath KW, Covington JK et al. Barriers to patient-clinician collaboration in asthma management: the patient experience. Asthma 2010; 47: $192-197$

27 Wilson SR, Strub P, Buist AS et al. Shared treatment decision making improves adherence and outcomes in poorly controlled asthma. Am J Respir Crit Care Med 2010; 181: 566-577

28 Haskard KB, Banta JE, Williams SL et al. Binge drinking, poor mental health, and adherence to treatment among California adults with asthma. J Asthma 2008; 45: 369-376

29 Petro $W$. Verbesserung der Compliance in der Therapie des Asthma bronchiale - Wie ist die Evidenz und wie kann man den Nutzen messen? Dtsch Med Wochenschr 2009; 134: S394-S397

30 Holgate S, Bigaard H, Bjemer C et al. The Brussels Declaration: the need for change in Asthma management. Eur Respir J 2008; 32: 1433 - 1442

31 Halbert RJ, Tinkelman DG, Globe DR et al. Measuring asthma control is the first step to patient management. J Asthma 2009; 46: 659-664

32 Joshi AV, Madhavan SS, Ambegaonkar A. Association of medication adherence with workplace productivity and healthrelated quality of life in patients with asthma. J Asthma 2006; 43: 521-526

33 van Dulmen S, Sluijs E, van Dijk L et al. Patient adherence to medical treatment: a review of reviews. BMC Health Services Research 2007; 7: $55-68$

34 Bosch-Capblanch X, Abba K, Prictor M et al. Contracts between patients and healthcare practitioners for improving patients adherence to treatment, prevention and health promotion activities. Cochrane Database Syst Rev 2007: CD004808

35 Janson SL, McGrath KW, Covington JK et al. Individualized asthma selfmanagement improves medication adherence and markers of asthma control. J Allergy Clin Immunol 2009; 123: 840-846

36 Gamble J, Stevenson M, Heaney LG. A study of multilevel interventions to improve non-adherence in difficult to control asthma. Respir Med 2011; 105: 1308-1315

37 Bender BG, Rand C. Medication nonadherence and asthma treatment cost. Curr Opin Allergy Clin Immunol 2004; 4: 191-195

38 Morisky DE, Green LW, Levine DM. Concurrent and predictive validity of a self reported measure of medication adherence and longterm predictive validity of blood pressure control. Med Care 1986; 24: 67-74

39 Osterberg $L G$, Rudd P. Medication adherence for antihypertensive therapy. In: Oparil S, Weber MA (eds) Hypertension: a comparison to Brenner and Rector's The Kidney. 2nd ed. Philadelphia: Elsevier Mosby; 2005: 848 
40 Mühlig S, Bergmann $\mathrm{K}-\mathrm{CH}$, Twesten $\mathrm{O}$ et al. Therapiemitarbeit bei ambulanten Asthmapatienten: Empirischer Vergleich der Complianceraten bei unterschiedlicher Operationalisierung der MedikamentenCompliance. Pneumologie 2001; 55: 177-189

41 Bartlett SJ, Krishnan JA, Rickert KA. Maternal depressive symptoms and adherence to therapy in Inner-City children with Asthma. Pediatrics 2004; 113: 229-237

42 Stilley CS, Bender CM, Dunbar-Jacob J et al. The impact of cognitive function on medication management: three studies. Health Psychol 2010; 29: 50-55

43 Bender BG. Risk Taking, depression, adherence, and symptom control in adolescents and young adults with asthma. Am J Crit Care Med 2006; 173: 953 -957

44 Starobin D, Bargutin M, Rosenberg I et al. Asthma control and compliance in a cohort of adult asthmatics: First survey in Israel. IMAJ 2007; 9: $358-360$

45 Horne R. Adherence to asthma medication: a question of ability? Prim Care Respir J 2011; 20: 118 - 119

46 Gilberg K, Lavari M, Wade $S$ et al. Analysis of medication use patterns: Apparent overuse of Antibiotics and underuse of prescription drugs for Asthma, Depression and CHF. J Managed Care Pharm 2003; 3: 232 237

47 Gillissen A. Patients adherence in Asthma. J Physiol Pharmacol 2007; 58: $205-222$

48 Schneider A, Biessecker K, Quinzler R et al. Higher preference for participation in treatment decisions is associated with lower medication adherence in asthma patients. Patient Educ Couns 2007; 67: 57-62

49 Maziak W, von Mutius E, Keil $U$ et al. Predictors of health care utilization of children with Asthma in the community. Pediatr Allergy Immunol 2004; 15: 166 - 171

50 Roy A, Battle $K$, Lursluchachai $L$ et al. Inhaler device, administration technique and adherence to inhaled corticosteroids in patients with asthma. Prim Care Respir J 2011; 20: 148 - 154

51 Jentzsch NS, Camargos PA. Methods of assessing adherence to inhaled corticosteroids therapy in children and adolescents. Adherence rates and their implications for clinical practice. J Bras Pneumol 2008; 34: 614-621

52 DiMatteo MR. Variations in patients' adherence to medical recommendations: A quantative review of 50 years of research. Med Care 2004; 42: $200-209$

53 Giraud V, Roche N. Misuse of corticosteroid metered-dose inhaler is associated with decreased asthma stability. Eur Respir J 2002; 19 : $246-251$

54 Morisky DE, Ang A, Krousel-Wood M et al. Predictive Validity of a Medication Adherence Measure for Hypertension Control. J Clin Hypertension 2008; 10 : $348-354$

55 Haynes RB, Ackloo E, Sahota $N$ et al. Interventions for enhancing medication adherence. Cochrane Database Syst Rev 2008; 2: CD000011

56 Bender B, Milgrom H, Rand C. Nonadherence in asthmatic patients: is there a solution to the problem? Ann Allergy Asthma Immunol 1997; 79: $177-186$
57 Lind A, Kaplan L, Berg GD. Evaluation of an Asthma Disease Management Program in a Medicaid Population. Dis Manage Health Outcomes 2006; $14: 151-161$

58 Stock S, Redaelli M, Luengen $M$ et al. Asthma: prevalence and cost of illness. Eur Respir J 2005; 25: 47-53

59 Nowak D, Volmer T, Wettengel R. Asthma bronchiale - eine Krankheitskostenanalyse. Pneumologie 1996; 50: 364 - 371

60 Schramm B, Ehlken B, Smala A et al. Cost of illness of atopic asthma and seasonal allergic rhinitis in Germany: 1-yr retrospective study. Eur Respir J 2003; 21: $116-122$

61 Demoly P, Annunziata K, Gubba E et al. Repeated cross-sectional survey of patient-reported asthma control in Europe in the past 5 years. Eur Respir Rev 2012; 21: 66 - 74

62 Demoly P, Gueron B, Annunziata $K$ et al. Update on asthma control in five European countries: results of a 2008 survey. Eur Respir Rev 2010; 19: $150-157$

63 Gibson PG, Powell H. Written action plans for asthma: an evidencebased review of the key components. Thorax 2004; 59: 94-99

64 Price $D$, Wolfe $S$. Delivery of asthma care: patients' use of and views on healthcare services as determined from a nation-wide interview survey. Asthma J 2000; 5: 141 - 144

65 Haughney J, Barnes G, Partridge $M$ et al. The Living \& Breathing Study: a study of patients' views of asthma and its treatment. Prim Care Respir J 2004; 13: $28-35$

66 Meng YY, Leung KM, Berkbigler D et al. Compliance with US asthma management guidelines and specialty care: a regional variation or national concern? J Eval Clin Pract 1999; 5: 213-221

67 Van Lente EJ, Willenborg P, Egger B. Auswirkungen der Disease-Management-Programme auf die Versorgung chronisch kranker Patienten in Deutschland - eine Zwischenbilanz. Gesundheits- und Sozialpolitik 2008; 62: $10-18$

68 Petro W, Schulenburg JM, Greiner W et al. Effizienz eines Disease Management Programmes bei Asthma. Pneumologie 2005; 59: 101 - 107

69 Ofman JJ, Badamgarav E, Henning J. Does disease management improve clinical and economic outcomes in patients with chronic diseases? A systematic review. American Journal of Medicine 2004; 117: 182 - 192

70 Mattke S, Seid M, Sai M. Evidence for the effect of disease management: Is US\$ 1 billion a year a good investment? Am J Managed Care 2007; 13: $670-676$

71 Fireman B, Bartlett J, Selby J. Can disease management reduce health care costs by improving quality? Health Affairs 2004; 23: 63-75

72 Sidorov J, Shull $R$, Tomcavage J et al. Does diabetes disease management save money and improve outcomes? Sponsored disease management program among patients fulfilling health employer data and information set criteria A report of simultaneous short-term savings and quality improvement associated with a health maintenance organization Diabetes Care 2002; 25: 684-689

73 Villagra VG, Ahmed T. Effectiveness of a disease management program for patients with diabetes. Health Affairs 2004; 23: 255-266

74 Dasgupta R, Guest JF. Factors affecting UK primary-care costs of managing patients with asthma over 5 years. Pharmacoeconomics 2003; 21: $357-369$ 